\title{
The innate immune repertoire in Cnidaria - ancestral complexity and stochastic gene loss David J Miller ${ }^{\star *}$, Georg Hemmrich ${ }^{\star \dagger}$, Eldon E Ball ${ }^{\ddagger}$, David C Hayward ${ }^{\ddagger}$, Konstantin Khalturin ${ }^{\dagger}$, Noriko Funayama§, Kiyokazu Agata ${ }^{\S}$ and Thomas CG Bosch ${ }^{\dagger}$
}

\begin{abstract}
Addresses: *ARC Centre of Excellence in Coral Reef Studies and Comparative Genomics Centre, James Cook University, Townsville, Queensland 4811, Australia. 'Zoological Institute, Christian-Albrechts-University Kiel, Olshausenstrasse, 24098 Kiel, Germany. ${ }^{\ddagger}$ ARC Centre for the Molecular Genetics of Development, Research School of Biological Sciences, Australian National University, Canberra ACT 2601, Australia. §Department of Biophysics, Kyoto University, Kitashirakawa-Oiwake, Sakyo-ku, Kyoto 606-8502, Japan.
\end{abstract}

$\ltimes$ These authors contributed equally to this work.

Correspondence: Thomas CG Bosch. Email: tbosch@zoologie.uni-kiel.de

Published: 16 April 2007

Genome Biology 2007, 8:R59 (doi:10.1 I86/gb-2007-8-4-r59)

The electronic version of this article is the complete one and can be found online at http://genomebiology.com/2007/8/4/R59
Received: 2 November 2006

Revised: 22 December 2006

Accepted: 16 April 2007

(C) 2007 Miller et al.; licensee BioMed Central Ltd.

This is an open access article distributed under the terms of the Creative Commons Attribution License (http://creativecommons.org/licenses/by/2.0), which permits unrestricted use, distribution, and reproduction in any medium, provided the original work is properly cited.

\begin{abstract}
Background: Characterization of the innate immune repertoire of extant cnidarians is of both fundamental and applied interest - it not only provides insights into the basic immunological 'tool kit' of the common ancestor of all animals, but is also likely to be important in understanding the global decline of coral reefs that is presently occurring. Recently, whole genome sequences became available for two cnidarians, Hydra magnipapillata and Nematostella vectensis, and large expressed sequence tag (EST) datasets are available for these and for the coral Acropora millepora.
\end{abstract}

Results: To better understand the basis of innate immunity in cnidarians, we scanned the available EST and genomic resources for some of the key components of the vertebrate innate immune repertoire, focusing on the Toll/Toll-like receptor (TLR) and complement pathways. A canonical Toll/TLR pathway is present in representatives of the basal cnidarian class Anthozoa, but neither a classic Toll/TLR receptor nor a conventional nuclear factor (NF)- $\mathrm{KB}$ could be identified in the anthozoan Hydra. Moreover, the detection of complement $\mathrm{C} 3$ and several membrane attack complex/perforin domain (MAC/PF) proteins suggests that a prototypic complement effector pathway may exist in anthozoans, but not in hydrozoans. Together with data for several other gene families, this implies that Hydra may have undergone substantial secondary gene loss during evolution. Such losses are not confined to Hydra, however, and at least one MAC/PF gene appears to have been lost from Nematostella.

Conclusion: Consideration of these patterns of gene distribution underscores the likely significance of gene loss during animal evolution whilst indicating ancient origins for many components of the vertebrate innate immune system. 


\section{Background}

The innate immune system is the first line of defense against pathogens, and in non-chordates is assumed to be the sole means by which any non-self cells are detected and either killed or contained [1]. Innate immunity in vertebrates is essentially a two-tier system consisting on one hand of phagocyte activation by the interaction of specialized surface receptors with pathogens or pathogen-derived components, and on the other of the direct opsonization and lysis of pathogens via the complement cascade. Whilst the vertebrate innate immune system has been the subject of intense investigation and is relatively well understood, studies of invertebrate immunity, which have focused primarily on the arthropods Drosophila and various horseshoe crab species [2-4], have revealed some striking similarities. For example, in both Drosophila and vertebrates, the Toll/Toll-like receptor (TLR) mediates the activation of appropriate response genes to microbial challenge $[5,6]$.

Toll and the TLRs are transmembrane proteins with a characteristic domain structure consisting of an extracellular amino-terminal domain containing leucine-rich repeats (LRRs) responsible for pattern recognition and an intracellular Toll interleukin receptor (TIR) domain that mediates signal transmission. Although the Toll and TLR families of arthropods and mammals are thought to have independently diversified $[7,8]$, all Tolls and TLRs signal via a common pathway that is conserved between Drosophila and mammals. The ultimate step in this pathway is translocation of nuclear factor (NF)- $\mathrm{BB}$ or its fly counterpart (the Dif/Rel heterodimer) into the nucleus, where it stimulates transcription of appropriate response genes. The immune repertoire of the horseshoe crab Carcinoscorpius includes a complex complement pathway that has both opsonic and lytic effector functions [9]. Horseshoe crab complement $\mathrm{C}_{3}$ is functionally homologous with mammalian $\mathrm{C}_{3}$, mediating phagocytosis of bacteria (by hemocytes) in a strikingly similar manner.

Whilst these specific studies imply that at least some innate immune mechanisms have been conserved, broader comparative studies highlight the extent of gene loss and divergence in various metazoan lineages. For example, although Carcinoscorpius clearly uses a vertebrate-like complement system, none of the central components of the cascade $\left(\mathrm{C}_{2}, \mathrm{C}_{3}, \mathrm{C}_{4}\right.$, $\mathrm{C}_{5}$ ) are encoded by the genomes of the ecdysozoans Drosophila, Caenorhabditis or Anopheles. Moreover, the sole Toll/TLR in Caenorhabditis elegans and C. brigssae is not known to function in the context of immunity, nor does that reported in the horseshoe crab Tachypleus tridentatus [10]. There are also important differences between the Toll/TLR systems of Drosophila and mammals. For example, some mammalian TLRs themselves act as pattern recognition receptors (PRRs) upon microbial challenge, whereas in fly this is not the case [11]. Moreover, whereas most of the ten or so vertebrate TLRs function primarily in immunity, only one of the nine fly (and ten mosquito) Tolls functions in this con- text. The others play a role in development [10], most famously in controlling differentiation in the dorsal/ventral axis.

The significance of gene loss in animal evolution has recently been brought into focus by preliminary expressed sequence tag (EST) and genomic analyses of some 'basal' animals (Figure 1), particularly the anthozoan cnidarians Acropora millepora and Nematostella vectensis $[12,13]$ and the planarian Dugesia japonica [14]. Paradoxically, the genomes of these morphologically simple animals contain many genes previously thought to have evolved much later in the context of vertebrate complexity, and most of the complexity of signaling pathways and transcription factors associated with higher animals is represented in the anthozoan datasets [13,15-17]. In contrast to Drosophila and Caenorhabditis, which have undergone substantial gene loss, for at least some groups of genes Acropora and Nematostella appear to have preserved much of the genetic complexity of the common metazoan ancestor. For example, whereas fly and worm have each lost approximately half of the ancestral Wnt complement, all but one of the 12 known Wnt subfamilies is represented in Nematostella [15]. The emerging cnidarian EST and genomic datasets are, therefore, potentially highly informative with respect to the ancestral immunological repertoire. In addition to this basic evolutionary significance, understanding the bases of cnidarian immunity is of major applied significance in light of the dramatic decline in coral health that has occurred on a global scale over the past 20 years. Increasing human activity in coastal zones throughout the world has led to declines in water quality with increased sediment, nutrient and heavy metal concentrations. These have all had detrimental effects on corals with an associated increase in the prevalence of disease, and perhaps led to some new diseases, although this is less certain, as coral diseases are very poorly understood. Most are named for their symptoms, for example, 'white band disease', 'black band disease', and 'rapid wasting syndrome' and causative agents are frequently unknown. In the face of this uncertainty it is important that coral defense mechanisms should be better understood.

Although cnidarians have no specialized immune cells, at least some display highly specific allorecognition characteristics. Allorecognition, xenorecognition, and killing mechanisms have been demonstrated in several hydrozoans [18-20] and anthozoans [21-23]. Allorecognition is thought to protect colonial cnidarians from fusion with genetically different individuals and to prevent germ line parasitism. The effector mechanisms range from contact avoidance involving chemical sensing, to barrier formation, or usage of nematocysts. For example, the sea anemone Anthopleura xanthogrammmica will 'tolerate' adjacent clonal individuals, but will attempt to 'reject' heterogenic clones with which it comes into contact $[24,25]$. In the anthozoans, Stylophora pistillata and Montipora verrucosa branches within one colony will readily fuse while branches of genetically different individuals never 


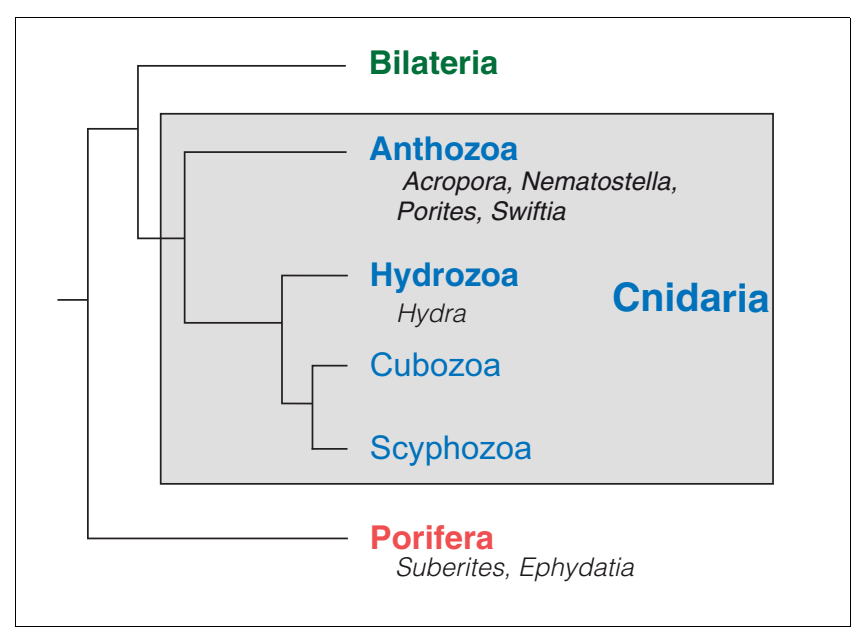

Figure I

Relationships at the base of the Metazoa. Cnidarians are amongst the simplest animals at the tissue grade of organization, and are often regarded as the closest outgroup to the Bilateria. Within the Cnidaria, the class Anthozoa is basal, whereas the Hydrozoa is derived. The sponges (Porifera) are unquestionably animals, but represent a lower level of organization. The affinities and relationships of genera mentioned in the text are indicated.

undergo fusion $[22,26,27]$. Fusion of two conspecific individuals is occasionally referred to as 'natural transplantation'. Observations in sea anemones (Anthopeura elegantissima, Phymactis clematis) and gorgonians (Eunicella stricta) indicate that individual colonies possess unique sets of histocompatibility elements, which are recognized as nonself by all other conspecific colonies [23,28]. In Hydrozoa, the same phenomenon was reported for Millepora dichotoma [29] and studied in great detail in the colonial marine hydroid Hydractinia echinata [30]. Hydractinia in fact was among the first invertebrates shown to display a genetically based system of intolerance against allogenic tissue: for more than 50 years it has been known that allorecognition and the inability to fuse stolons of different colonies is under the control of one polymorphic gene [31,32]. Recent efforts using defined genetic lines of the hydroid Hydractinia symbiolongicarpus have confirmed this and shown that the single chromosomal region contains at least two loci [33].

The availability of whole genome sequences of two basal cnidarians at respectably high levels of redundancy - the hydrozoan Hydra magnipapillata ( $>6$-fold coverage) and the anthozoan Nematostella vectensis (>7.6-fold coverage [17]) together with large-scale EST datasets for these and for the coral Acropora millepora potentially offer new perspectives on the origins of mammalian immune functions. Here we report the results of a screen of the available genomic and EST resources for the cnidarian counterparts of key components of the vertebrate innate immune repertoire.

\section{Results \\ The toll receptor and other proteins containing the TIR domain}

Searching the Hydra predicted protein collection using the available hidden Markov models (HMMs) identified only four TIR domain-containing proteins, two of which are clearly related to MyD88, which functions downstream of TLRs (Table 1). Consistent with their assignment as MyD88 family members, both of these Hydra proteins also contain the characteristic DEATH domain. The two other Hydra TIR proteins are atypical transmembrane proteins in having relatively short extracellular domains that are devoid of the LRR domains that characterize Toll and the TLRs (Figure 2). cDNAs encoding these proteins have previously been isolated by the Bosch laboratory (unpublished data) and their functions are presently under investigation; these proteins are known as HyTRR-1 and HyTRR-2. Surprisingly, extensive searching of the Hydra genome and all available EST/cDNA resources failed to identify any proteins having the canonical Toll/TLR structure, characterized by possession of both LRR and TIR domains.

Whereas only four TIR proteins are present in Hydra, substantially more could be identified amongst the predicted proteins from Nematostella using HMM-based search methods. Five of them were sufficiently complete to be included in the analyses presented here. These include a single MyD88 homolog (NvMyD88) and a protein (NvTLR-1) clearly related to members of the Toll/TLR family (Figure 2). Whereas the mammalian TLRs, and some members of the fly Toll/TLR family, have only a carboxy-terminal cysteine-rich motif flanking the LRRs proximal to the membrane, Nematostella NvTLR-1 is predicted to contain both carboxy- and aminoterminal-flanking cysteine-rich motifs in the extracellular part of the protein (Figure 2). This suggests that fly and anemone Toll more closely reflect the ancestral domain structure than do the mammalian TLRs. Moreover, a phylogenetic analysis (Figure 3 ) groups the TIR in Nematostella NvTLR-1 with its fly and human counterparts, with strong bootstrap support. Surprisingly, three more of the predicted Nematostella TIR proteins also contain multiple immunoglobulin (Ig) domains (Figure 2), and thus reflect the domain structure of mammalian interleukin 1 receptors (IL-1Rs). NvIL-1R1 and NvIL-1R2 each contain three Ig domains, and NvIL-1R3 contains two predicted Ig domains (Figure 2) but may be incomplete. In the phylogenetic analysis based on TIR domains the Nematostella IL-1R-like proteins form a clade distinct from both the MyD88 and Toll/TLR types (Figure 3), although these cnidarian TIRs appear to be distinct from those in the vertebrate IL-1Rs (data not shown). Several other TIR proteins were detected amongst the sequences of Nematostella (Additional data file 1), but were not subjected to further analysis as the TIR domains were incomplete or the sequences were judged likely to be artifactual. Two complete TIRs were identified by searching the available coral datasets. The trace archive yielded one TIR from Acropora palmata (ApGe- 
Table I

Overview of innate immunity components present or absent in selected Cnidaria

\begin{tabular}{|c|c|c|c|c|c|c|c|c|c|}
\hline & \multicolumn{6}{|c|}{ Anthozoa } & \multicolumn{3}{|c|}{ Hydrozoa } \\
\hline & \multicolumn{3}{|c|}{ Nematostella } & \multicolumn{3}{|c|}{ Acropora } & \multicolumn{3}{|c|}{ Hydra } \\
\hline & & Accession no. & e-value & & Accession no. & e-value & & Accession no. & e-value \\
\hline \multicolumn{10}{|c|}{ TLR pathway } \\
\hline LBP & + & gnl|ti|| | 39929806 & $7 e-51$ & ND & & & + & gb|DT619160 & $2 e-13$ \\
\hline CDI4 & - & & & ND & & & - & & \\
\hline TLR & + & 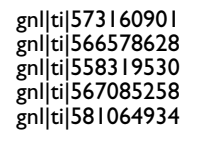 & le-47 & + & gb|EF090256 & $2 e-7$ & - & & \\
\hline MyD88 & + & gnl|ti|| I 39972660 & $4 e-26$ & ND & & & + & $\mathrm{gb} \mid \mathrm{CV} 182656$ & le- 18 \\
\hline IRAK & + & gn||ti|| | 46 | | 969 | & $3 e-14$ & ND & & & + & gb|DT608600 & $2 e-10$ \\
\hline TRAF6 & + & gnl|ti|| | 35509399 & $2 e-51$ & + & gb|DY583।89 & le-38 & + & gb|CV985667 & $3 e-4 I$ \\
\hline TAKI & + & gnl|ti|| | 35635219 & $|e-5|$ & + & gb|DY583694 & $8 e-119$ & + & gb|DN8| 2953 & le-45 \\
\hline IKK & + & gnl|ti|| | 35636054 & $5 e-68$ & ND & & & + & $\mathrm{gb} \mid C V 985420$ & $2 e-60$ \\
\hline$N F-\kappa B$ & + & gnl|ti|| I 39960940 & le-74 & + & gb|DY58297। & $3 e-36$ & - & & \\
\hline \multicolumn{10}{|c|}{ IFN pathway } \\
\hline TRAM & + & gnl|ti|| | 39940977 & $9 e-66$ & + & gb|DY579224 & $5 e-72$ & + & gb|DT6I5400 & le-58 \\
\hline TRIF & + & gnl|ti|| | 39933368 & $4 e-07$ & ND & & & $?$ & & \\
\hline TBK-I & ? & & & ND & & & $?$ & & \\
\hline IRF3 & + & gnl|ti|| | $46 \mid 21907$ & $6 e-13$ & ND & & & + & gb|DT6095।8 & $2 \mathrm{e}-14$ \\
\hline p65 & - & & & ND & & & - & & \\
\hline IFN- $\beta$ & - & & & ND & & & - & & \\
\hline \multicolumn{10}{|c|}{ ECSIT pathway } \\
\hline ECSIT & + & gnl|ti|| | 39978500 & $4 e-35$ & ND & & & + & $\begin{array}{l}\text { gnl||ti|| } 223628 \\
732\end{array}$ & $2 e-18$ \\
\hline MEKKI & + & gn||ti|| | 39956887 & $2 \mathrm{e}-28$ & + & $\mathrm{gb|DY} 581 \mid 38$ & $3 e-83$ & + & $\begin{array}{l}\text { gnl|ti|| } 226566 \\
543\end{array}$ & $3 e-25$ \\
\hline MKKs & + & gnl|ti|557758729 & $\mathrm{Ie}-14$ & ND & & & + & $\begin{array}{l}\text { gnl|ti||I|2|9|8 } \\
104\end{array}$ & $1 \mathrm{e}-18$ \\
\hline
\end{tabular}


Table I (Continued)

Overview of innate immunity components present or absent in selected Cnidaria

\begin{tabular}{|c|c|c|c|c|c|c|c|c|c|}
\hline JNK & + & gnl|ti|l I 35503269 & le- 106 & ND & & & + & $\begin{array}{l}\text { gn||ti|8773345 } \\
88\end{array}$ & $2 e-33$ \\
\hline p38 & + & gnl|ti|| | $399590 \mid 4$ & |e-1 14 & + & gb|DY5797/2 & $5 e-111$ & + & $\begin{array}{l}\mathrm{gnn}|| \mathrm{ti} \mid 6860485 \\
04\end{array}$ & $7 e-39$ \\
\hline API & + & gn||ti|| I 39792930 & $3 e-10$ & + & gb|DY581320 & $3 e-09$ & + & $\mathrm{gb} \mid \mathrm{CX} 771032$ & $7 e-10$ \\
\hline ATF & + & gn||ti|| | 39796564 & $4 e-11$ & ND & & & + & $\mathrm{gb} \mid \mathrm{CN} 624618$ & $3 e-06$ \\
\hline
\end{tabular}

Other TLR related proteins

HyTRR-I

HyTRR-2

ILI-R related proteins

$\begin{array}{lcccc}\text { ILIR-I } & + & \text { gnl|ti|573|82253 } & 0 & \text { ND } \\ \text { ILIR-2 } & + & \text { gnl|ti|557993643 } & 0 & \text { ND } \\ \text { ILIR-3 } & + & \text { gnl|ti|567060226 } & 0 & \text { ND }\end{array}$

Complement system related

proteins

C3/A2M related

$\mathrm{C} 6 / \mathrm{C} 7 / \mathrm{C} 8$

MAC/PF domain-containing

proteins

Apextrins

Tx60-A

MPEG
ND

ND

$\begin{array}{ll}+ & \text { gb|DQ449929 } \\ + & \\ +\quad \text { gb|DQ449930 } & 0\end{array}$

$+\quad$ gb|EF091848 6e-15 + gb|CVI 85005
gb|DT613346
gb|CF655657
gb|DT620043

gnl|ti|ll39936806 7e-48 + gb|DY579588 9e-48 gb|DY579588 $7 e-48$
$3 e-35$

gb|CV464226 gb|CD680300 gb|BP512716 gb|Bर12716 gb|DN2468I।
$4 e-04$

le-07 


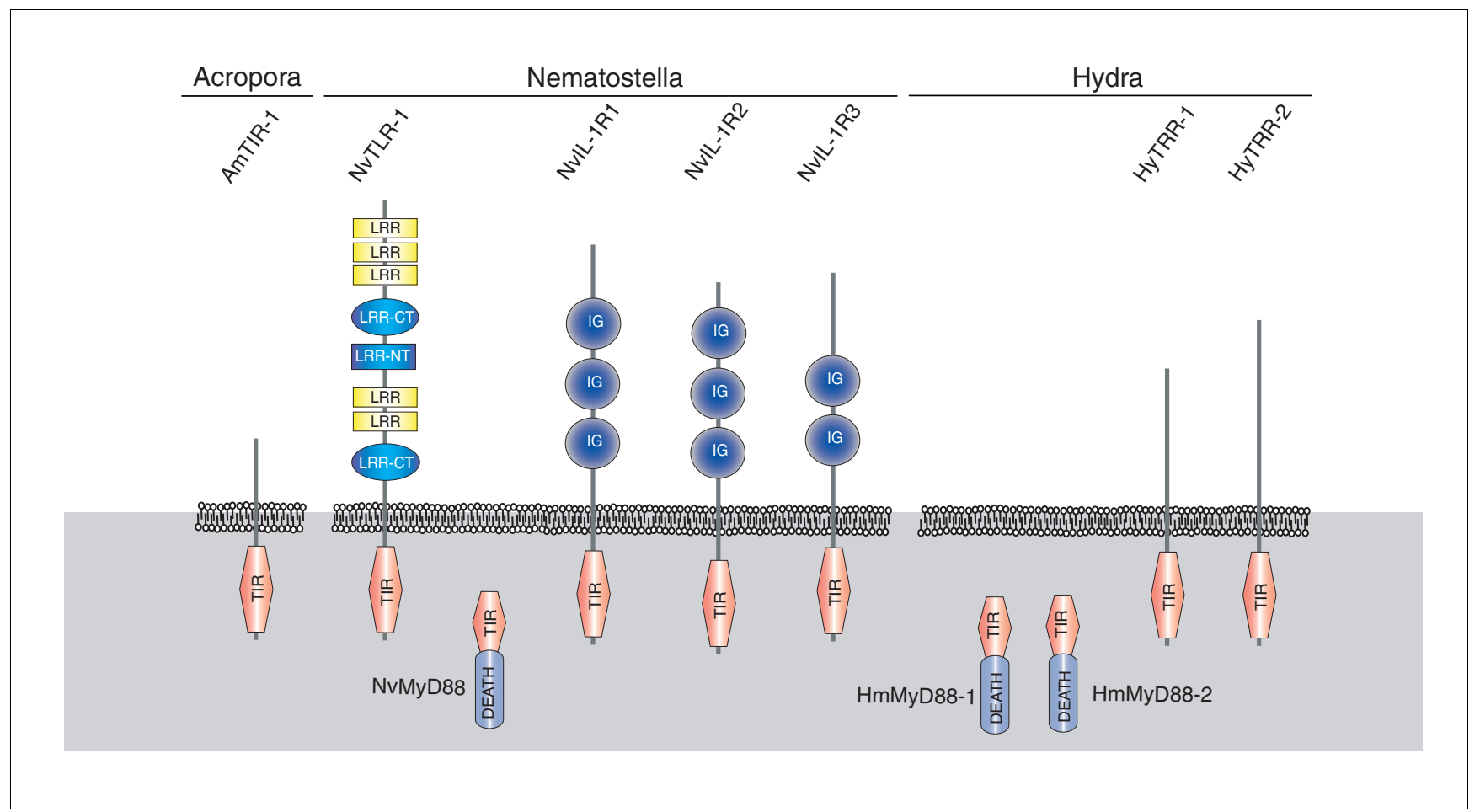

Figure 2

Summary of domain structures of TIR domain-containing proteins identified in selected Cnidaria.

nomic) and a second was encoded by an A. millepora EST (AmTIR-1). These two coral TIRs are most similar to those in the Nematostella IL-1R-like proteins (Figure 3), but no linked domains have yet been identified in these cases.

The Müller group recently reported the identification of MyD88 in a demosponge, Suberites domuncula [34]. However, whilst phylogenetic analyses clearly grouped the TIR in this sponge sequence with those present in unambiguous MyD88 orthologs (Figure 3), domain searching indicates that the predicted sponge protein may not have a functional DEATH domain.

\section{The Toll/TLR pathway is ancestral but some components are missing or highly divergent in Hydra} Most of the intracellular mediators of Toll/TLR signaling could be identified in Nematostella and Acropora, but some key components appear to have either been lost or diverged beyond recognition in Hydra (Table 1). The absence of a Toll/ TLR protein sensu stricto from Hydra is discussed above, but in addition only a single highly derived Rel domain could be found in Hydra whereas unambiguous NF- $\kappa \mathrm{B}$ homologs are present in both Nematostella and Acropora (Table 1). In addition to the pathway leading to nuclear localization of NF$\kappa \mathrm{B}$, Toll/TLR signaling can activate the Jun N-terminal kinase (JNK) and p38 mitogen-activated protein kinase (MAPK) pathways, leading to transcription of a range of target genes via the AP1 (activating protein 1)/ATF (activating transcriptionfactor 1) factors. Toll/TLR signaling via JNK/ MAPK requires the participation of the ECSIT (evolutionarily conserved signaling intermediate in Toll pathways) adaptor protein [35], which also provides a link between the Toll/TLR and TGF-b (transforming growth factor-beta)/BMP (bone morphogenic protein) pathways [36]. The presence of ECSIT as well as the key components of the JNK/MAPK pathway in the cnidarian datasets (Table 1, Figure 4) indicates an early origin for this variant of Toll/TLR signaling. The discovery of a conserved predicted ECSIT coding sequence in the fresh water sponge Ephydatia fluviatilis (N Funayama, personal observation) additionally supports this view.

Wiens et al. [34] have suggested that a sponge-specific cell surface protein known as SLIP (sponge LPS-interacting protein) functions as a pattern recognition receptor and effectively substitutes for Toll/TLR in antimicrobial defence. The sponge MyD88-related protein nominally functions downstream of SLIP in the proposed pathway [34]. In support of the idea that sponges lack Toll/TLRs, the authors cite a lack of TLRs in a screen of 15,000 ESTs. As sponge 'MyD88' and SLIP are co-immunoprecipitated by the reciprocal antibodies [34], they clearly can interact in vitro. Some TLR pathway components could also be identified in the available sponge data, but the equivocal status of the sponge MyD88 related protein means that it is unclear at this time whether sponges have a canonical Toll/TLR pathway. 


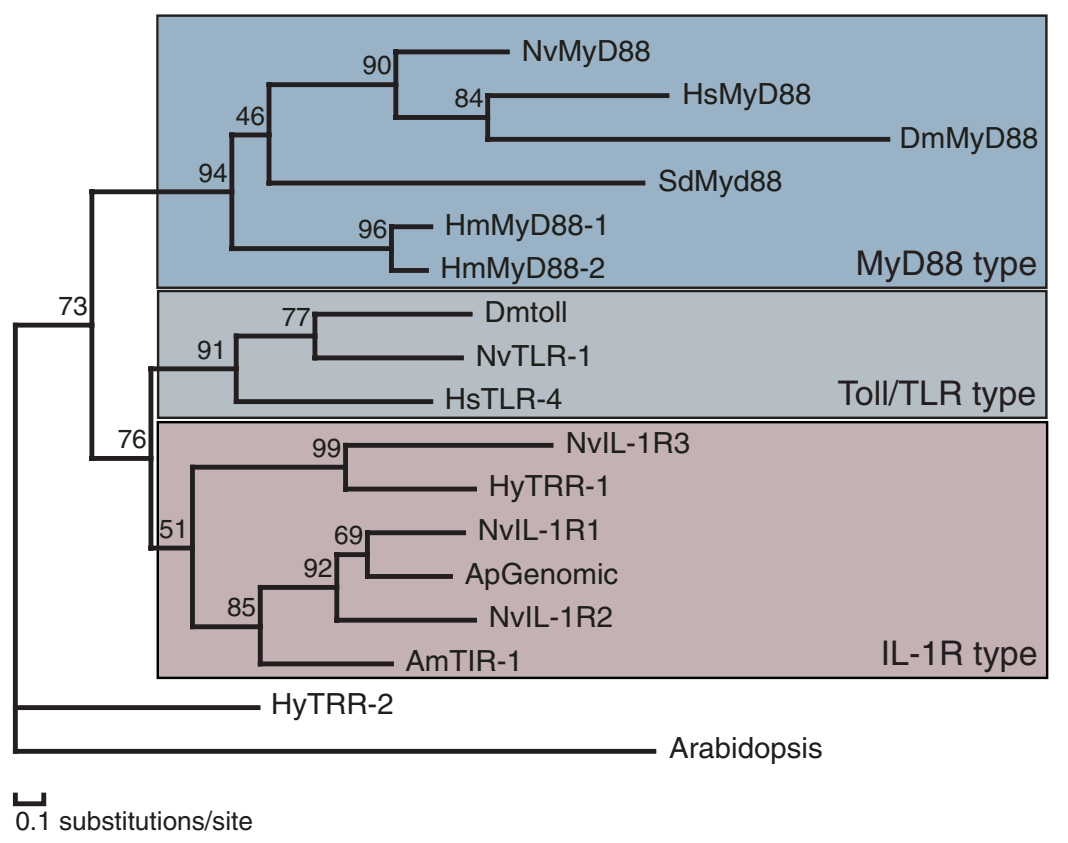

\section{Figure 3}

Phylogenetic analysis of cnidarian TIR sequences in comparison to a selection of TIR domains from other species. The maximum likelihood (ML) tree shown is the result of analysis of an HMM-based alignment of TIR domains. A number of the TIR sequences identified and discussed in the text are incomplete due to the presence of introns of unknown size, and hence were not included in the phylogenetic analyses. Three clades are resolved by these analyses, corresponding to the TIR domains characteristic of the 'MyD88-type', 'Toll/TLR-type' and 'IL-IR-type'. In addition to the TIR domain, the first of these types contains a death domain and the second contains multiple LRRs. Like the mammalian receptors for interleukin I, the three Nematostella proteins falling into the third clade each also contain multiple immunoglobulin domains. Note that HyTRRI does not contain such domains and that it is not yet clear whether either of the Acropora proteins does. The Acropora sequences included in the analysis were predicted from A. palmata genomic clones (ApGenomic) and from an A. millepora cDNA clone (AmTIR-I). Hydra lacks a canonical Toll/TLR, having only two MyD88 genes and the two sequences known as TRR-I and TRR-2; H. magnipapillata and $\mathrm{N}$. vectensis sequences are indicated by the prefixes $\mathrm{Hy}$ and $\mathrm{Nv}$, respectively. Reference sequences: HsMyD88, human MyD88 (SwissProt:Q99836); DmMyD88, fly MyD88 (GenBank:AAL56570); SdMyD88, Suberites MyD88 (EMBL:CAI68016); Dmtoll, fly Toll (SwissProt:P08953); HsTLR4, human TLR4 (EMBL:CAD99|57); Arabidopsis (GenBank:AAN289|2).

\section{Cnidarian complement $\mathrm{C} 3$ and related proteins}

The complement component $\mathrm{C}_{3}$ has recently been reported in another anthozoan cnidarian, the octocoral Swiftia [37], and the corresponding gene has recently been cloned from Acropora (Hayward, unpublished data). The Acropora $\mathrm{C}_{3}\left(\mathrm{C}_{3}-\right.$ Am) gene is first expressed strongly in the endoderm of the planula as it elongates following gastrulation (Figure $5 \mathrm{a}$ ). The endodermal expression is not uniform, being most intense in a subset of dark staining cells that have not yet been characterized. As the planula elongates expression becomes somewhat weaker, with the strongest expression localized to the aboral endoderm (Figure 5b). Post-settlement (Figure 5c-e) expression is limited to the endoderm and is particularly strong in the endoderm of the polyp as it rises from the calcifying platform at its base (for example, Figure $5 \mathrm{~d}$ ).

C3 has a complex domain structure. Whilst anthozoan $\mathrm{C}_{3} \mathrm{~s}$ resemble their deuterostome counterparts both in domain structure (Figure $5 \mathrm{f}$ ) and sequence, not only could no corresponding gene be identified in Hydra, but also some of the domains characteristic of $\mathrm{C}_{3}$ (ANATO, C $345 \mathrm{C}$; Figure $5 \mathrm{f}$ ) could not be detected in any Hydra protein. Although lacking a canonical $\mathrm{C}_{3}$, Hydra contains a gene encoding A2M related domains. Interestingly, in situ hybridization in Hydra using a probe covering these typical A2M-related domains (Figure $5 \mathrm{f}$; A2M-comp/A2M-recep) showed expression restricted to the endodermal epithelium (Figure $5 \mathrm{~g}$ ), as was the case with Acropora $\mathrm{C}_{3}$.

\section{MAC/PF domain containing proteins in Cnidaria}

Searching for other components of the complement cascade, we identified proteins containing a membrane attack complex/perforin domain (MAC/PF) similar to that present in complement component $\mathrm{C} 6$ and related proteins. HMM searching identified just two MAC/PF domain-containing proteins in Hydra (Table 1), whereas four proteins were identified in Nematostella. Two MAC/PF proteins were also identified amongst the Acropora ESTs. Database searches and analyses of predicted domain structures revealed that most of the cnidarian MAC/PF sequences are likely to fall into three 
groups corresponding to the known proteins types MPEG, TX-6oA and apextrin (Table 1, Figure 5h).

TBlastN-based searches of the Nematostella genome identified a gene matching strongly to the human macrophage expressed protein 1 (MPEG1; GenBank:XP 166227) and its abalone homolog abMPEG1 (GenBank:AAR82936) [38]. A clearly related gene in $S$. domuncula has recently been implicated as an effector in a hypothetical sponge innate immune defence pathway [34]. Recombinant Suberites MPEG has anti-bacterial activity against Gram-negative bacteria, and is up-regulated after lipopolysaccharide (LPS) treatment [34]. The MPEG1 family clearly has an ancient evolutionary history (the sponge and human sequences have $28 \%$ identity and $46 \%$ similarity) [34] but only in Suberites has any functional characterization been done. Despite the presence of MPEG1 in the sponge and an anthozoan, no corresponding gene could be identified in Hydra.

The nematocyst venom of at least some anthozoans contains the protein TX-60A [39], and two of the Nematostella MAC/ PF proteins and one of the Acropora ESTs clearly correspond to this protein type (Table 1). TX-60A has an epidermal growth factor (EGF) domain immediately carboxy-terminal of the MAC/PF domain. In Hydra, this domain structure can be found in Hy-MAC, one of the two Hydra MAC/PF proteins (Figure $5 \mathrm{~h}$, Table 1). However, it is unclear whether the Hydra and anthozoan sequences are orthologous, as overall sequence identity is low. In situ hybridization analysis shows that expression of Hy-MAC is restricted to gland cells that are interspersed throughout the endoderm of Hydra (Figure 5i). Since endodermal gland cells and nematocysts are terminally differentiated [40], this pattern of expression is not easy to reconcile with a common function for the venom TX-60A and Hy-MAC.

\section{Apextrin, a gene lost from Nematostella}

The third class of cnidarian MAC/PF proteins represented in the Hydra and Acropora ESTs (Figure 5h) contains no identifiable domains other than MAC/PF. These proteins have moderate overall similarity to the echinoderm apextrins $[41,42]$ and to the apicomplexan protein family to which the Plasmodium membrane attack ookinete protein (MAOP) [43] belongs. MAOP is responsible for rupture of epithelial cells in the insect host by the ookinete stage of the parasite. Surprisingly, apextrin seems to be a case of gene loss from Nematostella as, despite clearly related genes being present in Hydra and Acropora, extensive searching of both the predicted protein collection and the anemone genome using a variety of tools failed to identify an apextrin-related gene (Table 1).

To explore the significance of this case of apparent gene loss, the expression pattern of an apextrin gene was examined by in situ hybridization in Acropora (Figure 5k-o). Apextrin-Am expression first appears in scattered ectodermal cells at the oral (blastopore) end of the embryo as it begins to elongate following blastopore closure (Figure $5 \mathrm{k}$ ). As the embryo continues to elongate expression increases in intensity and the zone of expression spreads toward the aboral end, initially still in scattered cells (Figure 5l), but as elongation continues apparently in all ectodermal cells (Figure $5 \mathrm{~m}$ ), as is clear in transverse section (Figure $5 \mathrm{n}$ ). As the planula settles, expression becomes less obvious at the oral end, eventually becoming limited to a belt marking the transition between the tissue that was formerly aboral (bottom), and the end bearing the oral pore, which subsequently forms the mouth of the polyp (Figure 50). These expression data suggest that the primary function of apextrin-Am is in the ectoderm leading up to metamorphosis; hence, secondary loss of the corresponding gene from Nematostella may be explicable in terms of the very different modes of development of these two animals (see Discussion).

In adult Hydra, whole mount in situ hybridization showed an expression of the apextrin-like gene in groups of ectodermal cells arising from the interstitial cell lineage (Figure $5 \mathrm{j}$ ), which may reflect a possible functional shift in an organism where metamorphosis is absent.

In addition to the above, the Nematostella dataset yielded MAC/PF domain-containing proteins having high similarity to the neural cell adhesion molecule spondin-1 (Additional data file 1).

\section{Discussion}

These preliminary analyses of the newly available genomic and EST datasets indicate that a surprising number of key components of the innate immune system, including the Toll/ TLR pathway and some complement cascade components, were in place at the base of the Eumetazoa. Also represented in the datasets are proteins strongly matching both the tumor necrosis factor (TNF) and Nod-like receptors and with the same domain structures (data not shown).

The analyses presented here are consistent with the idea of a genetically complex common metazoan ancestor [12,13]; clearly the Toll/TLR, MyD88 and IL-1R protein families were distinct prior to the divergence of the Cnidaria from the Bilateria, and a Toll/TLR pathway may predate even the Porifera/ Eumetazoa split. The discovery of proteins with the same domain structure as the IL-1R in Nematostella indicates that this receptor type predates chordate origins and that its original ligands may not have been interleukins. However, as the TIR domains in the cnidarian IL-1R-like and mammalian IL$1 \mathrm{R}$ proteins are divergent, separate evolutionary origins cannot yet be ruled out despite the similarities at the level of domain structure.

It is also likely that a prototypic complement effector pathway involving $\mathrm{C}_{3}$ and multiple $\mathrm{MAC} / \mathrm{PF}$ proteins was in place in 


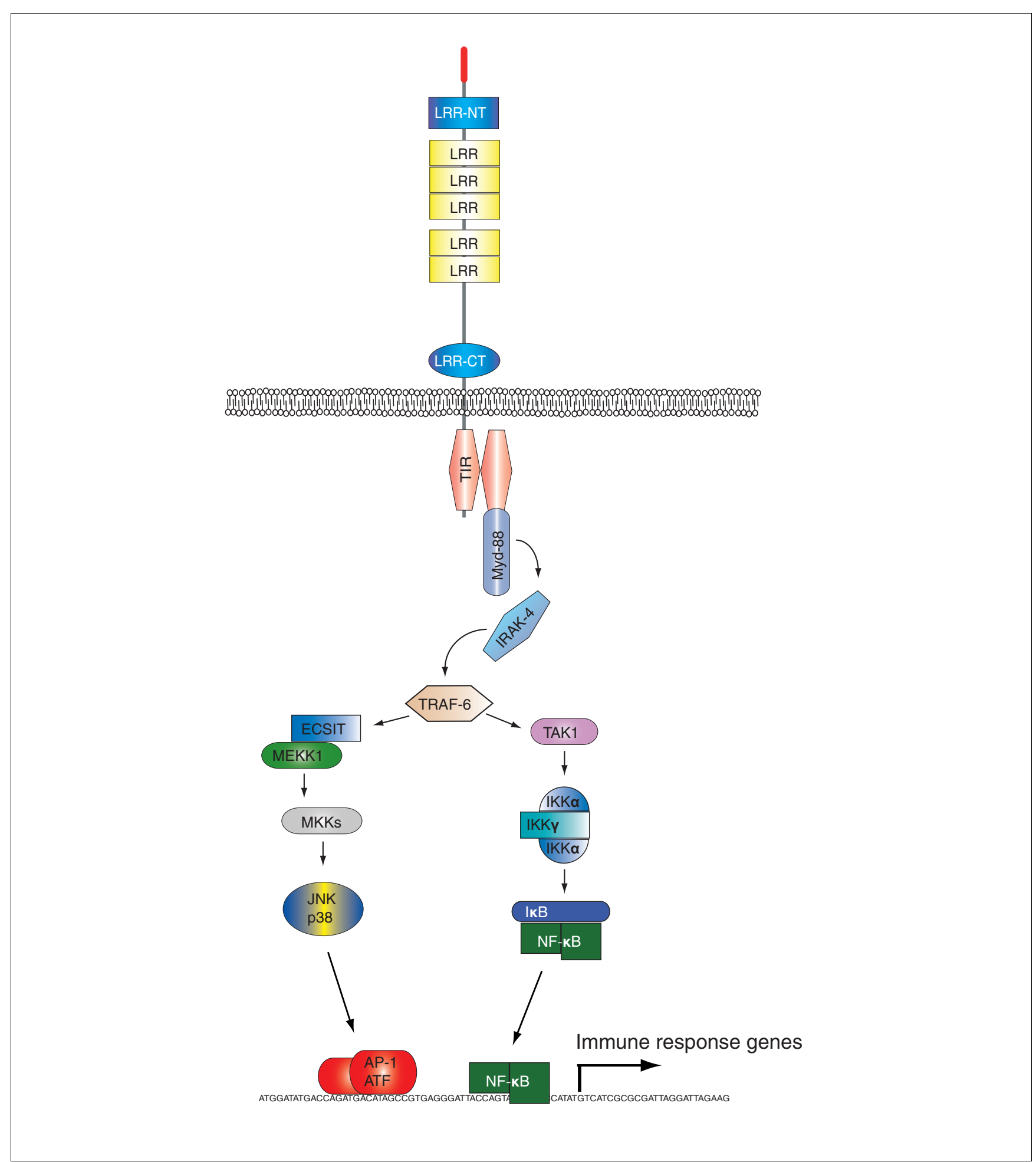

\section{Figure 4}

Signaling pathways downstream of the Toll/TLRs. Pattern recognition, either indirectly or directly, by Toll/TLRs results in activation of NF-KB (vertebrates) or the Dif/Rel heterodimer (Drosophila) and thus transcription of appropriate immune response genes. At TRAF6, the classic Toll/TIR pathway (shown in the right branch) is linked to the JNK/p38 pathway (shown in the left branch) by the ECSIT protein, which acts as a regulator of MEKK-I processing [35]. Components of both pathways downstream of Toll/TLRs are represented in the cnidarian datasets (Table I). ECSIT may also act as a link between these and the TGF-b signaling pathway, since it forms complexes with BMP-pathway restricted Smads and is essential for regulation of the BMP-target gene TIx2 [36]. All of the components of the TGF-b signaling pathway are also known from anthozoan cnidarians [I3]. 


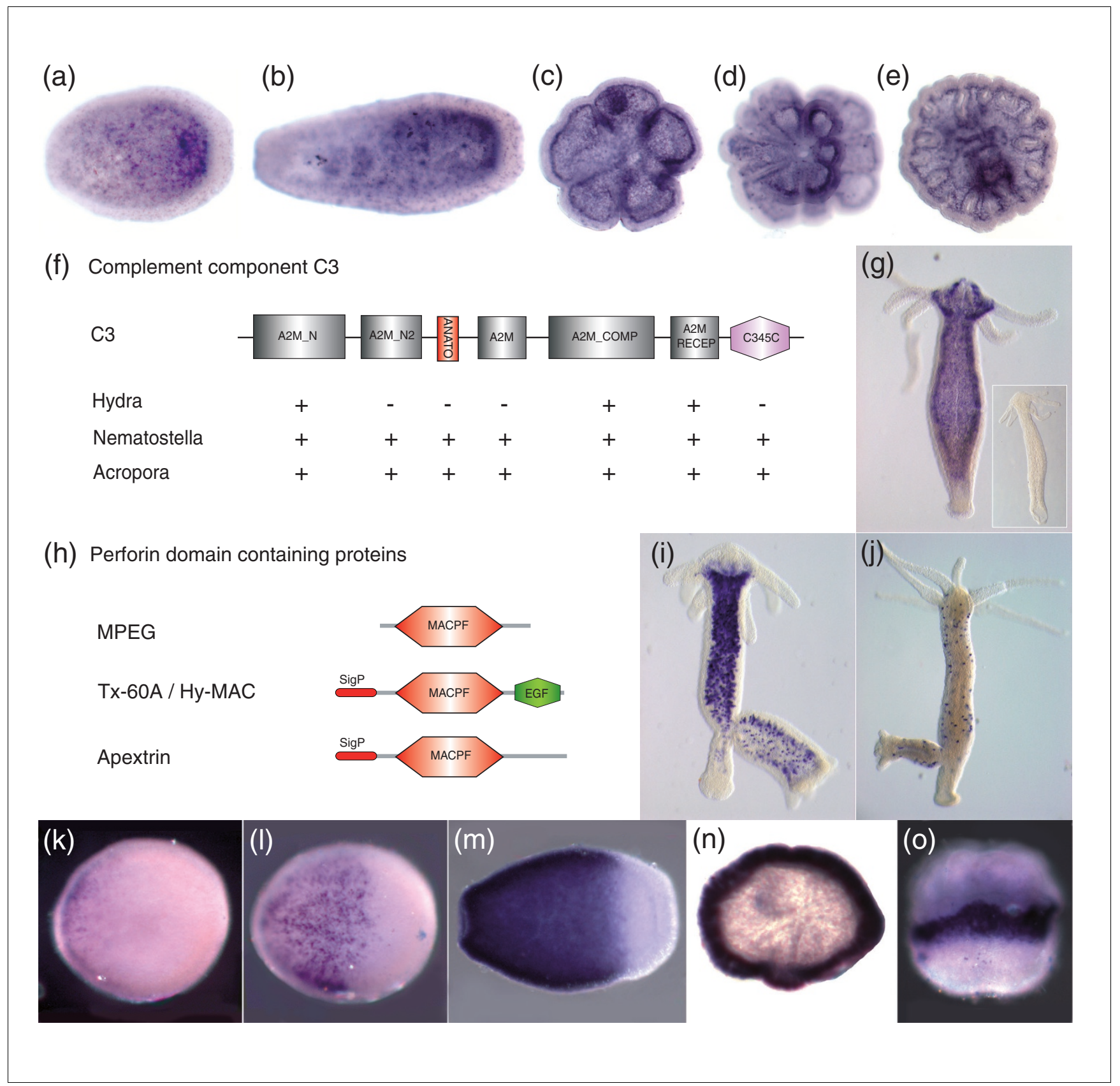

\section{Figure 5}

Complement component C3 and MAC/PF domain-containing proteins in Cnidaria. (a-e) In situ hybridization of C3-Am in Acropora. Expression first becomes apparent in scattered endodermal cells concentrated at the aboral end as the planula elongates from sphere to pear (a) and eventually to spindle (b). Endodermal expression continues post-settlement (c-e), becoming especially strong in the upper part of the polyp as it rises from the calcifying base (d). Post-settlement, the polyp consists of a series of hollow chambers interconnected beneath the mouth. The line of strong staining peripherally is the result of viewing the endoderm vertically, while elsewhere one is looking through the staining layer. (f) Domain map and presence $(+) /$ absence $(-)$ data for the various protein domains characteristic of complement $\mathrm{C} 3$ components in the Hydra, Nematostella and Acropora datasets. (g) In situ hybridization of the H. magnipapillata A2M-related gene. Hydra A2M-related transcripts are present in the endoderm along the whole body axis. Note that this Hydra gene lacks several of the C3-diagnostic domains that are present in the anthozoan C3s (see text). (h) Domain maps of major cnidarian MAC/PF proteins types. (i) Hydra Tx-60a in situ. The insert shows the sense control. (j) Hydra apextrin in situ. (k-o) Acropora apextrin in situ. Expression is first apparent in scattered ectodermal cells orally as the planula begins to elongate $(k)$. At slightly later stages expression has spread toward the aboral end of the planula, still in scattered cells (I). As the elongation process continues, uniform strong expression is localized in all ectodermal cells in the oral two-thirds of the planula $(\mathrm{m})$. The strong ectodermal expression is clearly apparent in this transilluminated transverse section cut from the central region of the planula $(\mathrm{n})$. Following settlement, expression continues at the oral end of the planula, before frequently becoming limited to a narrow ring separating oral and aboral tissue (o). 
Ureumetazoa. Comparisons of the TIR and MAC/PF complements of Hydra and Nematostella highlight the likely extent of gene loss and sequence divergence in the former - not only does Hydra appear to have lost the Toll-receptor, but NF- $\mathrm{BB}$ has also either been lost or has diverged beyond recognition. Moreover, only a few TIR proteins could be identified in Hydra compared to the rich representation of these in the anthozoans. In addition, Hydra appears to have lost a number of MAC/PF proteins and lacks an equivalent of the ancestral complement $\mathrm{C}_{3}$ protein, implying that although anthozoans most likely have a prototype complement effector pathway, this has undergone degeneration in Hydra. Hydra is an efficient producer of a number of potent antimicrobial peptides (Bosch, unpublished data), indicating that at least some of the functions of the complement effector system may have been subsumed by pathways involved in synthesis of these peptides.

The observation that genes of two of the three classes of cnidarian immune-repertoire genes examined here are expressed in the endoderm (Figure 2) was unexpected, but is consistent with the primary sites of expression of such genes in many animals. In Drosophila, the fat body (an endodermal derivative, and the functional homolog of the mammalian liver) is the predominant source of the antimicrobial peptides whose synthesis is under the control of the Toll and Imd pathways [44] and in Caenorhabditis the gut is the primary source of antimicrobials $[45,46]$. Although mammalian skin contains immune sensory cells (Langerhans cells, dendritic cells and so on), these are also endodermal derivatives, hence there is a deep evolutionary link between the immune system and the endoderm.

Other specific cases of gene loss from Hydra have been documented [47] (Seneca, unpublished data), hence there are precedents for the apparent loss of immune repertoire components reported here. More surprising is the implication that gene losses may also have occurred in Nematostella, as evidenced by the apparent absence of the apextrin gene that is present in both Hydra and Acropora. This loss may be associated with differences between Nematostella and Acropora during the planula to polyp transition. Metamorphosis from the motile planula to the sessile polyp involves dramatic tissue remodeling [48] and gene expression changes in Acropora (Grasso, unpublished data), and the massive up-regulation of apextrin leading up to and during metamorphosis (Figure 5k-o) is consistent with a role in this process. It is worthy of note that apextrin is expressed in the tissue that is least remodeled at the time of metamorphosis. By contrast, it appears that there are no dramatic events comparable to Acropora metamorphosis occurring during this same period of Nematostella development; rather, Nematostella undergoes a simple and continuous transition from planula to polyp and appears to show some neotenous features, continuing to glide over the bottom aboral end first for some time after metamorphosis [49], and never forming a pedal disc [50]. Thus, loss of this gene may be related to the different modes of development of these two animals. This comparison between two anthozoans with different modes of development is clearly reminiscent of the original identification of apextrin as a gene specifically expressed in the 'direct' developing seaurchin Heliocidaris erythrogramma during metamorphosis, but which is not expressed during the development of $H$. tuberculata, a congeneric 'indirect' developer [41,42]. Because indirect development is considered to be ancestral in sea urchins, these authors hypothesized that apextrin had been coopted to function in metamorphosis in $H$. erythrogramma. The specific ectodermal expression of homologous proteins during metamorphosis in a cnidarian and an echinoderm is either a remarkable example of convergence or reflects conservation of function at some level. This latter possibility carries with it the implication that the 'simple' continuous planula to polyp transition seen in Nematostella might represent a derived state, and that the more dramatic metamorphosis seen in Acropora might more closely reflect the ancestral condition.

\section{Conclusion}

An important general implication that flows from these data is that gene loss may occur stochastically. If the genes in a pathway function only in that pathway, then one might expect that entire pathways would disappear following loss of one key component. However, the Hydra data appear to contradict this; most of the intracellular intermediates of Toll/TLR signaling are present despite loss of all of the major receptor types (both Toll/TLR and the IL-1R types known from more basal cnidarians are apparently absent from Hydra). Moreover, simple comparisons between related animals (for example, Nematostella versus Acropora) are unlikely to be informative in terms of understanding the origins of genes [50] - loss of a gene from Nematostella and loss of a specific function in the indirect developing urchin seems more likely than the independent co-option of the same protein to related roles in Acropora and a direct developing urchin. Stochastic loss underlying the distribution patterns of genes across the Metazoa may account for some cases of assumed lateral gene transfer - chance retention of an ancestral gene in one or a few animal lineages might easily be confused with lateral transfer. Reconstructing the genome of the common animal ancestor will not, therefore, be a simple undertaking; it will require whole genome data for a wide range of 'lower' as well as 'higher' animals.

\section{Materials and methods Datasets}

Genomic and EST sequence data were downloaded from public databases at NCBI (dbEST, Trace archive) or originate from private investigators (D Miller, K Agata) and were stored on a local comparative genomics analysis platform [51]. The raw datasets included 10,171,402 genomic reads and 163,221 
ESTs for $H$. magnipapillata, 5,996,730 genomic reads and 146,976 ESTs for $N$. vectensis, 14,625 genomic reads and 10,232 ESTs for $A$. millepora, 11,025 genomic reads for $A$. palmata and 11,450 genomic reads for Porites lobata. A set of 36,820 ESTs from the fresh water sponge $E$. fluviatilis is maintained in the lab of K Agata. For the corals A. millepora, A. palmata and $P$. lobata only pilot genomic sequencing data are available to date. Genomic trace data were maintained in the original raw format, whereas ESTs were clustered and assembled using TGICL tools from TIGR [52]. The ESTscan software [53] was used to infer unigene and predicted peptide sequences from the assembled ESTs.

\section{Database search, sequence analysis and phylogenetic methods}

For database searches a local Blast-platform [51] and the public Blast platform at NCBI [54] were used. Domain searches were performed using SMART [55] and a local install of HMMer [56]. Profile HMMs for the investigated domains were obtained from PFAM [57] and Superfamily [58] databases. Seqtools [59] and BioEdit [60] were used for general sequence analysis. Protein sequence alignments were created using ClustalW [61] and the HMMalign script included in the HMMer package. Maximum likelihood phylogenetic analyses were undertaken using MolPhy version 2.3 [62] using the Dayhoff substitution matrix and local rearrangement search mode.

\section{In situ hybridization}

Whole mount in situ hybridization on Hydra polyps was performed as previously described by Grens et al. [63]. Acropora embryos were fixed and processed for in situ hybridization as described in de Jong et al. [64] following the detailed in situ protocol given in Hayward et al. [65]. For all genes shown, sense controls gave no hybridization signals.

\section{Additional data files}

The following additional data are available with the online version of this paper. Additional data file lists accession numbers for additional sequences identified within the database searches that were not further characterized in the present study.

\section{Acknowledgements}

This work was supported by Grants from the Deutsche Forschungsgemeinschaft (DFG/SFB6 I7) to TCGB and from the Australian Research Council (ARC) directly to DJM and EEB (Grants A0010543I, DP0209460 and DP0344483) and via both the Centre for the Molecular Genetics of Development and the Centre of Excellence for Coral Reef Studies. The authors thank H Bode, R Steele and D Rokshar for their efforts in heading the Hydra and Nematostella Genome projects.

\section{References}

I. Beutler B: Innate immunity: an overview. Mol Immunol 2004, 40:845-859.

2. Litman GW, Cannon JP, Dishaw LJ: Reconstructing immune phy- logeny: new perspectives. Nat Rev Immunol 2005, 5:866-879.

3. Royet J, Reichhart JM, Hoffman JA: Sensing and signaling during infection in Drosophila. Curr Opin Immunol 2005, I 7: I I- I7.

4. Flajnik MF, Du Pasquier L: Evolution of innate and adaptive immunity: Can we draw a line? Trends Immunol 2004, 25:640-644.

5. Lemaitre B, Reichhart JM, Hoffman JA: Drosophila host defense: differential induction of antimicrobial peptide genes after infection by various classes of microorganisms. Proc Natl Acad Sci USA 1997, 94:146|4-14619.

6. Medzhitov R, Preston-Hurlburt P, Janeway CA Jr: A human homologue of the Drosophila Toll protein signals activation of adaptive immunity. Nature 1997, 388:394-397.

7. Rock FL, Hardiman G, Timans JC, Kastelein RA, Bazan JF: A family of human receptors structurally related to Drosophila Toll. Proc Natl Acad Sci USA 1998, 95:588-593.

8. Luo C, Zheng L: Independent evolution of Toll and related genes in insects and mammals. Immunogenetics 2000, 51:92-98.

9. Zhu Y, Thangamani S, Ho B, Ding JL: The ancient origin of the complement system. EMBO / 2005, 24:382-394.

I0. Inamori K, Ariki S, Kawabata S: A toll-like receptor in horseshoe crabs. Immunol Rev 2004, I98: I06-I I5.

II. Michel T, Reichhart JM, Hoffmann JA, Royet J: Drosophila Toll is activated by Gram-positive bacteria through a circulating peptidoglycan recognition protein. Nature 200I, 4I 4:756-759.

12. Kortschak RD, Samuel G, Saint R, Miller DJ: EST analysis of the cnidarian, Acropora millepora, reveals extensive gene loss and rapid sequence divergence in the model invertebrates. Curr Biol 2003, 13:2190-2195.

13. Technau U, Rudd S, Maxwell P, Gordon PM, Saina M, Grasso LC, Hayward DC, Sensen CW, Saint R, Holstein TW, et al:: Maintenance of ancestral complexity and non-metazoan genes in two basal cnidarians. Trends Genet 2005, 21:633-639.

14. Mineta K, Nakazawa M, Cebria F, Ikeo K, Agata K, Gojobori T: Origin and evolutionary process of the CNS elucidated by comparative genomics analysis of planarian ESTs. Proc Natl Acad Sci USA 2003, 100:7666-767I.

I5. Kusserow A, Pang K, Sturm C, Hrouda M, Lentfer J, Schmidt HA, Technau U, von Haeseler A, Hobmayer B, Martindale MQ, Holstein TW: Unexpected complexity of the Wnt gene family in a sea anemone. Nature 2005, 433:156-160.

16. Kamm K, Schierwater B: Ancient complexity of the non-Hox ANTP gene complement in the anthozoan Nematostella vectensis. Implications for the evolution of the ANTP superclass. I Exp Zoolog B Mol Dev Evol 2006, 306:589-596.

17. Ryan JF, Burton PM, Mazza ME, Kwong GK, Mullikin JC, Finnerty JR The cnidarian-bilaterian ancestor possessed at least 56 homeoboxes: evidence from the starlet sea anemone Nematostella vectensis. Genome Biol 2006, 7:R64.

18. Campbell RD, Bibb C: Transplantation in coelenterates. Transplant Proc 1970, 2:202-2II.

19. Leddy SV, Green DR: Historecognition in the Cnidaria. In Phylogenesis of Immune Functions Edited by: Warr GW, Cohen N. Boca Raton: CRC Press; 1991:103-116.

20. Bosch TCG, David CN: Immunocompetence in Hydra: Epithelial cells recognize self-nonself and react against it. I Exp Zool 1986, 238:225-234.

21. Hildemann WH, Bigger $\mathrm{CH}$, Johnston IS: Histoincompatibility reactions and allogeneic polymorphism among invertebrates. Transplant Proc 1979, I I:I I36-I | 42.

22. Hildemann WH, Jokiel PL, Bigger $\mathrm{CH}$, Johnston IS: Allogeneic polymorphism and alloimmune memory in the coral, Montipora verrucosa. Transplantation 1980, 30:297-301.

23. Lubbock $\mathrm{R}$ : Clone-specific cellular recognition in a sea anemone. Proc Natl Acad Sci USA 1980, 77:6667-6669.

24. Francis L: Clone specific segregation in sea anemone Anthopleura elegantissima. Biol Bull 1973, 144:64-72.

25. Sebens KP: Agonistic behaviour in the inter-tidal sea anemone Anthopleura xanthogrammica. Biol Bull 1984, 166:457-472.

26. Müller WE, Müller I, Zahn RK, Maidhof A: Intraspecific recognition system in scleractinian corals: morphological and cytochemical description of the autolysis mechanism. J Histochem Cytochem 1984, 32:285-288.

27. Chadwick-Furman NE, Rinkevich B: A complex allorecognition in a reef building coral: delayed responses, reversals and nontransitive hierarchies. Coral Reefs 1994, I 3:57-63.

28. Meinardi E, Florin-Christensen M, Paratcha G, Azcurra JM, FlorinChristensen J: The molecular basis of the self/nonself selectiv- 
ity of a coelenterate toxin. Biochem Biophys Res Commun 1995 , 2 1 6:348-354.

29. Frank U, Rinkevich B: Alloimmune memory is absent in the Red Sea hydrocoral Millepora dichotoma. J Exp Zool 2001, 291:25-29.

30. Frank U, Leitz T, Muller WA: The hydroid Hydractinia: a versatile, informative cnidarian representative. Bioessays 200I, 23:963-97I.

31. Hauenschild CV: Genetische und entwicklungsphysiologische Untersuchungen über Intersexualität und Gewebeverträglichkeit bei Hydractinia echinata. Flem Wilhem Roux' Archiv 1954, |47:|-4|.

32. Hauenschild CV: Über die Vererbung einer Gewebeverträglichkeitseigenschaft bei dem Hydroidpolypen Hydractinia echinata. Z Naturforsch |956, I I b:|32-|38.

33. Cadavid LF, Powell AE, Nicotra ML, Moreno M, Buss LW: An invertebrate histocompatibility complex. Genetics 2004, 1 67:357-365.

34. Wiens $M$, Korzhev M, Krasko A, Thakur NL, Perovic-Ottstadt S, Breter HJ, Ushijima H, Diehl-Seifert B, Muller IM, Muller WE: Innate immune defense of the sponge Suberites domuncula against bacteria involves a MyD88-dependent signaling pathway. Induction of a peforin-like molecule. J Biol Chem 2005, 280:27949-27959.

35. Kopp E, Medzhitov R, Carothers J, Xiao C, Douglas I, Janeway CA, Ghosh S: ECSIT is an evolutionarily conserved intermediate in the Toll/IL-I signal transduction pathway. Genes Dev 1999, | 3:2059-207|.

36. Xiao C, Shim JH, Kluppel M, Zhang SS, Dong C, Flavell RA, Fu XY, Wrana JL, Hogan BL, Ghosh S: Ecsit is required for BMP signaling and mesoderm formation during mouse embryogenesis. Genes Dev 2003, I 7:2933-2949.

37. Dishaw LJ, Smith SL, Bigger CH: Characterisation of a C3-like cDNA in a coral: phylogenetic implications. Immunogenetics 2005, 57:535-548

38. Mah SA, Moy GW, Swanson WJ, Vacquier VD: A perforin-like protein from a marine mollusk. Biochem Biophys Res Comm 2004, 3 1 6:468-475.

39. Oshiro N, Kobayashi C, Iwanaga S, Nozaki M, Namikoshi M, Spring J, Nagai H: A new membrane-attack complex/perforin (MACl PF) domain lethal toxin from the nematocyst venom of the Okinawan sea anemone Actineria villosa. Toxicon 2004, 43:225-228

40. Bosch TCG: Symmetry breaking in stem cells of the basal metazoan Hydra. In Progress in Molecular and Subcellular Biology: Asymmetric Cell Division Edited by: Macieira-Coelho A. Heidelberg: Springer; 2006:61-78.

4I. Haag ES, Raff RA: Isolation and characterization of three mRNAs enriched in embryos of the direct-developing sea urchin Heliocidaris erythrogramma: evolution of larval ectoderm. Dev Genes Evol 1998, 208: 188-204.

42. Haag ES, Sly BJ, Andrews ME, Raff RA: Apextrin, a novel extracellular protein associated with larval ectoderm evolution in Heliocidaris erythrogramma. Dev Biol 1999, 21 I:77-87.

43. Kadota K, Ishino T, Matsuyama T, Chinzei Y, Yuda M: Essential role of membrane-attack protein in malarial transmission to mosquito host. Proc Natl Acad Sci USA 2004, I 0 I: I 63 I 0- I63 I 5.

44. Mylonakis E, Aballay A: Worms and flies as genetically tractable animal models to study host-pathogen interactions. Infect Immun 2005, 73:3833-384I.

45. Mallo GV, Kurz CL, Couillault C, Pujol N, Granjeaud S, Kohara Y, Ewbank J): Inducible antibacterial defense system in $C$. elegans. Curr Biol 2002, I2:1209-1214.

46. Millet AC, Ewbank J: Immunity in Caenorhabditis elegans. Curr Opin Immunol 2004, I 6:4-9.

47. Chourrout D, Delsuc F, Chourrout P, Edvardsen RB, Rentzsch F, Renfer E, Jensen MF, Zhu B, de Jong P, Steele RE, Technau U: Minimal protohox cluster inferred from bilaterian and cnidarian Hox complements. Nature 2006, 442:684-687.

48. Ball EE, Hayward DC, Reece-Hoyes JS, Hislop NR, Samuel G, Saint R, Harrison PL, Miller DJ: Coral development: from classical embryology to molecular control. Int J Dev Biol 2002, 46:67|-678.

49. Hand C, Uhlinger KR: The culture, sexual and asexual reproduction, and growth of the sea anemone Nematostella vectensis. Biol Bull 1992, 182:169-176.

50. Collins AG, Cartwright P, McFadden CS, Schierwater B: Phylogenetic context and basal metazoan model systems. Integr Comp
Biol 2005, 45:585-594.

51. Compagen [http://www.compagen.org]

52. Pertea G, Huang X, Liang F, Antonescu V, Sultana R, Karamycheva S, Lee $Y$, White J, Cheung F, Parvizi B, et al.: TIGR Gene Indices clustering tools (TGICL): a software system for fast clustering of large EST datasets. Bioinformatics 2003, 19:65 I-652.

53. Iseli C, Jongeneel CV, Bucher P: ESTScan: a program for detecting, evaluating, and reconstructing potential coding regions in EST sequences. Proc Int Conf Intell Syst Mol Biol 1999: I38-I48.

54. Altschul SF, Gish W, Miller W, Myers EW, Lipman DJ: Basic local alignment search tool. J Mol Biol 1990, 2 I 5:403-4I0.

55. Letunic I, Copley RR, Schmidt S, Ciccarelli FD, Doerks T, Schultz J, Ponting CP, Bork P: SMART 4.0: towards genomic data integration. Nucleic Acids Res 2004, 32:DI42-DI44.

56. HMMER [http://hmmer.janelia.org/]

57. Sonnhammer EL, Eddy SR, Birney E, Bateman A, Durbin R: Pfam multiple sequence alignments and HMM-profiles of protein domains. Nucleic Acids Res 1998, 26:320-322.

58. Gough J, Karplus K, Hughey R, Chothia C: Assignment of homology to genome sequences using a library of hidden Markov models that represent all proteins of known structure. J Mol Biol 200I, 3 I 3:903-919.

59. SEQtools [http://www.seqtools.dk]

60. Hall TA: BioEdit: a user-friendly biological sequence alignment editor and analysis program for Windows 95/98/NT. Nucleic Acids Symp Ser 1999, 41:95-98.

6I. Thompson JD, Higgins DG, Gibson TJ: CLUSTAL W: improving the sensitivity of progressive multiple sequence alignment through sequence weighting, position-specific gap penalties and weight matrix choice. Nucleic Acids Res 1994, 22:4673-4680.

62. Adachi J, Hasegawa M: MOLPHY version 2.3: program for molecular phylogenetics based on maximum likelihood. Comput Sci Monogr 1996, 28: I- I 50.

63. Grens A, Gee L, Fisher DA, Bode HR: CnNK-2, an NK-2 homeobox gene, has a role in patterning the basal end of the axis in hydra. Dev Biol 1996, 180:473-488.

64. de Jong DM, Hislop NR, Hayward DC, Reece-Hoyes JS, Pontynen PC, Ball EE, Miller DJ: Components of both major axial patterning systems of the Bilateria are differentially expressed along the primary axis of a 'radiate' animal, the anthozoan cnidarian Acropora millepora. Dev Biol 2006, 298:632-643.

65. Hayward DC, Catmull J, Reece-Hoyes JS, Berghammer H, Dodd H, Hann SJ, Miller DJ, Ball EE: Gene structure and larval expression of cnox $2 \mathrm{Am}$ from the coral Acropora millepora. Dev Genes Evol 200I, 2 I I:10-19. 